Letter to the Editor

\section{Supper-selective ACTH-stimulated adrenal vein sampling is necessary for detecting precisely functional state of various lesions in unilateral and bilateral adrenal disorders, inducing primary aldosteronism with subclinical Cushing's syndrome}

\section{Dear Sir;}

Hiraishi et al. [1] had recently reported that the prevalence of primary aldosteronism (PA), complicated with subclinical Cushing's syndrome (SCS), was 8 of 38 PA patients (21\%). They also reported that PA/SCS patients could be classified into various sub- types [1]. They concluded that hypercortisolemia is usually concordant with imaging studies, while hyperaldosteronemia is frequently discordant with CT images, in which micro-APA may coexist at ipsilateral, bilateral, or contralateral side [1].

It is well known that PA manifests biochemically and pathologically as several subtypes, with aldosteroneproducing adenoma (APA) and idiopathic hyperaldosteronism (IHA) comprising most cases. These 2 entities can be difficult to distinguish clinically, biochemically, radiologically and even by adrenal vein sampling (AVS), especially in the rare case of multiple adrenal adenomas, which may occur either unilaterally or bilaterally. It is important to differentiate between these subtypes because APA is potentially surgically correctable, while IHA is usually medically managed [2].

On the other hand, AVS can detect the laterality of hyperaldosteronism in those patients, while it is still

Masao Omura, Jun Saito, Yoko Matsuzawa and Tetsuo Nishikawa

Division of Endocrinology and Metabolism, Endocrinology and Diabetes Center, Department of Medicine, Yokohama Rosai Hospital, Yokohama 222-0036, Japan

Submitted: Aug. 17, 2011; Accepted Aug. 19, 2011 as EJ11-0210 Released online in J-STAGE as advance publication Sep. 8, 2011

Correspondence to: Tetsuo Nishikawa, Endocrinology and Diabetes Center, Yokohama Rosai Hospital, 3211 Kozukue-cho, Kohoku-ku, Yokohama City, Kanagawa 222-0036, Japan.

E-mail: tetsuon@yokohamah.rofuku.go.jp difficult to differentiate bilateral hypersecretion of bilateral aldosterone-producing adenomas (Blt-APAs) from that of IHA. We have developed a new method of supper-selective ACTH-stimulated adrenal vein sampling (SS-ACTH-AVS) in order to improve the technical examination for differential diagnosis [3]. Adrenal effluents were sampled at the central veins and at one or two tributaries of adrenal veins in each gland. SS-ACTH-AVS can precisely analyze the functional state of steroidogenesis in various portions of each adrenal gland. We can also detect some tiny lesion(s) by SS-ACTH-AVS, which is not visible in CT images. Moreover, it is possible to differentiate Blt-APAs from IHA and we can postulate the decision of surgical treatment, such as partial adrenalectomy. Thus, we should perform SS-ACTH-AVS especially in the cases demonstrating an existence of adrenal lesions such as unilateral and bilateral tumors, or even no tumor in both sides in PA [3].

Cases 2 \& 5 with PA/SCS, reported by Hiraishi et al. demonstrated bilateral adrenal hyperaldosteronemia [1]. We would like to emphasize that SS-ACTH-AVS, by which we can easily detect the portion of localized (APA) or diffuse adrenal lesions (IHA) in PA, should be performed in Hiraishi's Cases $2 \& 5$ with PA/SCS. Furthermore, there were reported an interesting cases, such as radiographic evidence of multiple adrenal nodules in unilateral or bilateral adrenal glands [4], like as the cases reported by Hiraishi et al. [1]. SS-ACTHAVS is strongly indicational for unilateral/bilateral adrenal disorders demonstrating multiple nodules. They also reported that the absolute value of aldosterone was employed for diagnostic criteria for laterality of hyperaldosteronemia in PA/SCS, since cortisol levels considerably differed in adrenal veins in PA/SCS [1]. We also suggested that we should use absolute values, rather than using the ratio of aldosterone to cortisol in AVS [5].

\section{Acknowledgements}

This work was partly supported by the Grant for Research on Intractable Diseases provided from the Japanese Ministry of Health, Labour and Welfare.

\section{Disclosure}

The authors report no financial or other relationships that might lead to a conflict of interest in this work. 


\section{References}

1. Hiraishi K, Yoshimoto T, Tsuchiya K, Minami I, Izumiya H, Sasano H and Hirata Y (2011) Clinicopathological features of primary aldosteronism associated with subclinical Cushing’s syndrome. Endocr J 58: 543-551.

2. Nishikawa T, Omura M, Satoh F, Shibata H, Takahashi K, Tamura N and Tanabe A (2011) Guidelines for the diagnosis and treatment of primary aldosteronism - The Japan Endocrine Society 2009 -. Endocr J 58: 711721.

3. Nishikawa T, Matsuzawa $\mathrm{Y}$, Saito $\mathrm{J}$ and Omura M
(2010) Editorial-Is it possible to extirpate cardiovascular events in primary aldosteronim after surgical treatment. Jpn Clinical Medicine 1: 21-23.

4. Nishikawa T (2006) How many distinct tumors can exit simultaneously in an unilateral adrenal gland, and are they hormonally active or inactive ? Clin Exp Nephrol 10: $162-163$.

5. Omura M, Sasano H, Fujikawa T, Tashiro Y, Yamaguchi $\mathrm{K}$ and Nishikawa T (2002) Unique cases of unilateral hyperaldosteronemia due to multiple adrenocortical micronodules, which can only be detected by selective adrenal venous sampling. Metabolism 51: 350-355. 